\title{
Jackknife and bootstrap inferential procedures for censored survival data
}

\begin{abstract}
Confidence interval is an estimate of a certain parameter. Classical construction of confidence interval based on asymptotic normality (Wald) often produces misleading inferences when dealing with censored data especially in small samples. Alternative techniques allow us to construct the confidence interval estimation without relying on this assumption. In this paper, we compare the performances of the jackknife and several bootstraps confidence interval estimates for the parameters of a log logistic model with censored data and covariate. We investigate their performances at two nominal error probability levels and several levels of censoring proportion. Conclusions were then drawn based on the results of the coverage probability study.
\end{abstract}

Keyword: Bootstrap; Censored data; Confidence interval; Coverage probability; Log logistic 